\title{
The design and performance of the ATLAS Inner Detector trigger for Run 2
}

\author{
Ondřej Penc* on behalf of the ATLAS Collaboration, \\ *Academy of Sciences of the Czech Republic, \\ Institute of Physics, Prague, CZ
}

\begin{abstract}
The design and performance of the ATLAS Inner Detector trigger algorithms running online on the High Level Trigger processor farm with the early LHC Run 2 data are discussed. The redesign of the ID trigger, which took place during the 2013-15 long shutdown, in order to satisfy the demands of the higher energy LHC Run 2 operation is described. The Inner Detector High Level Trigger algorithms are essential for nearly all trigger signatures within the ATLAS trigger. The performance of the tracking algorithms with the early Run 2 data for the different trigger signatures is presented, including the detailed timing performance for the algorithms running on the redesigned single stage ATLAS HLT Farm. A comparison with the Run 1 strategy is made and demonstrates the superior performance of the strategy adopted for Run 2.
\end{abstract}

\section{INTRODUCTION}

A FTER a two year upgrade that took place between 2013 and 2015, the LHC and all its experiments are going to extend their previous successes from the first period of their operation called Run 1. No doubt the greatest success of the ATLAS experiment was the observation of a new particle in the search for the Standard Model Higgs boson performed along with the CMS experiment in 2012 [1]. This discovery is a great success of particle physics that proposed the mechanism that suggested the existence of such a particle almost half a century ago in 1964 [2], [3]. To build on this success in Run 2 - the second operation period of the LHC - the hardware and software for the ATLAS detector was upgraded to perform efficiently in the new conditions.

In the second section the LHC upgrade is described and the ATLAS detector is introduced with emphasis on the Inner Detector. The Inner Detector Trigger architecture comparison between Run 1 and Run 2 is discussed in the third section. Optimisations for Run 2 are mentioned in the fourth section. The new tracking strategy for tau reconstruction for Run 2 is covered in the fifth section and the performance on the early Run 2 data is presented in the sixth section. The results are summarized in the last section.

\section{LHC UPGRADE AND ATLAS INNER DETECTOR}

Towards the end of Run 1, the LHC was routinely delivering instantaneous luminosities of $7.7 \times 10^{33} \mathrm{~cm}^{-2} \mathrm{~s}^{-1}$ with proton-proton collisions at $8 \mathrm{TeV}$ centre-of-mass energy. Following the upgrade, the centre-of-mass energy was increased to $13 \mathrm{TeV}$ and the luminosity is intended to be $2 \times 10^{34} \mathrm{~cm}^{-2} \mathrm{~s}^{-1}$. The parameters of the LHC after the upgrade are summarized in Table I. Almost twice the beam energy provides a new probe of high energy physics. It will bring the confirmation of the Standard Model at yet unexplored energy regime or a discovery of physics beyond the Standard Model. The increased luminosity allow the precision study of Standard Model particles such as the top quark and especially the Higgs boson.

TABLE I

COMPARISON OF THE LHC PARAMETERS AT THE END OF RUN 1 AND THE HIGHEST EXPECTED PARAMETERS AFTER THE UPGRADE FOR RUN 2.

\begin{tabular}{llll}
\hline Machine parameter & Run1 & Run2 & Unit \\
\hline Centre-of-mass energy & 8 & 13 & $\mathrm{TeV}$ \\
Luminosity & $7 \times 10^{33}$ & $2 \times 10^{34}$ & $\mathrm{~cm}^{-2} \mathrm{~s}^{-1}$ \\
Bunch crossing spacing & 50 & 25 & $\mathrm{~ns}$ \\
L1 input frequency & 20 & 40 & $\mathrm{MHz}$ \\
Mean pileup & $\sim 20$ & $\sim 40$ & interactions per crossing \\
\hline
\end{tabular}

The ATLAS is a general-purpose detector at LHC located at CERN [4]. The detector comprises of several subsystems (ordered outside in) such as muon spectrometer in magnetic field of toroidal superconducting magnet, hadronic and electromagnetic calorimeters and the Inner Detector (ID). The ID is a high precision tracking device surrounding the ATLAS interaction point of the LHC beams to detect charged particles - products of the proton-proton collisions. It is placed inside $2 \mathrm{~T}$ axial magnetic field provided by a solenoid that is essential for particle charge and momentum identification.

The ID, shown in Fig. 1, is a set of three complementary detectors at the center of the ATLAS detector. The Transition Radiation Tracker (TRT) which is based on xenon gas detector straws is the outermost subsystem of the ID. The straws are arranged in 73 layers in the barrel part and 160 layers in each endcap. The TRT has $R-\Phi$ resolution of $130 \mu \mathrm{m}$.

The rest of the subsystems are based on silicon semiconductors. The Semiconductor Tracker (SCT) is placed within the TRT. It is based on stereo-angled doublets of silicon microstrips arranged in four layers in barrel and 9 layers in each endcap. The $R-\phi$ resolution in this case is $17 \mu \mathrm{m}$.

The innermost part of the ID is based on hi-resolution silicon pixels. The pixel detector was upgraded with a new innermost pixel barrel layer for Run 2 called Insertable BLayer (IBL). It improves the impact parameter resolution, enhances vertex identification, and increases tracking robustness [2]. The Pixel Detector with the IBL has 4 barrel layers and 3 disks in each endcap. The $R-\Phi$ resolution is $10 \mu \mathrm{m}$. 


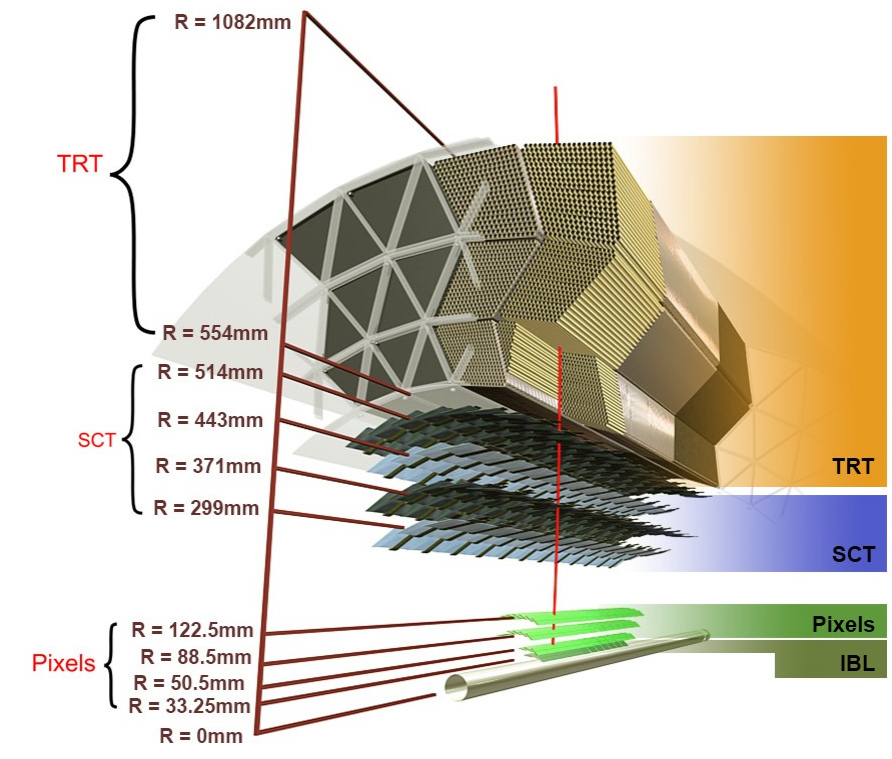

Fig. 1. Sketch of the ATLAS ID showing all its components, including the new IBL. The distances to the interaction point are also shown [5].

\section{RUN 1 VS. RUN 2 TRIGGER ARCHITECTURE}

Due to the LHC upgrade, the ATLAS trigger architecture had to undergo several changes to be able to fully exploit the new potential.

The time between bunch crossings was reduced from $50 \mathrm{~ns}$ to the $25 \mathrm{~ns}$ from the original design. This places a significant burden on the hardware and software subsystems of the ATLAS Trigger and lead to the trigger architecture upgrade. Also the high pileup multiplicity expected for Run 2 demands the need for a new tracking strategy and software optimisation. The CPU time consumption in simulated Run 2 events as a function of the pileup interaction multiplicity is illustrated in Fig. 2. Shown are the times for the two most time consuming stages of the Event Filter Inner Detector (EFID) trigger strategy as used during Run 1 . The time consumption clearly grows non-linearly with the pileup multiplicity indicating that this would quickly become too costly to run in the ATLAS High Level Trigger (HLT) at high multiplicities.

For the upgrade, the HLT architecture was simplified such that both the Run 1 software stages of the HLT - the Level 2 (L2) and Event Filter (EF) - have been merged into a single HLT stage to run on single CPU nodes. It simplifies the dataflow, removing the need for network communication between the L2 and EF trigger levels, and furthermore reduces requests to the DAQ system since common data preparation and storage was established.

The trigger processing still consists of similar algorithms as in the Run 1 [7]. It still works within the RoIs, but instead of two separate stages, L2 and subsequent EF where the processing started from scratch and repeated the CPU expensive pattern recognition on a different processing unit, the EF processing just becomes an additional step performed on the single HLT CPU node. Due to that the equivalent sequence of algorithms can be more efficiently combined, avoiding repetition and thus reducing reconstruction time [8].

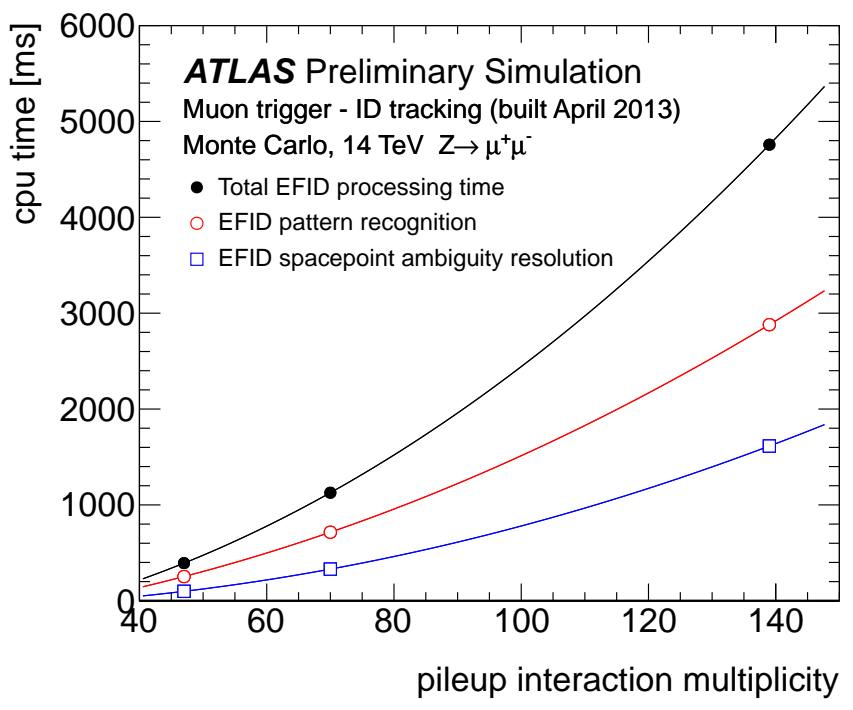

Fig. 2. Dependence of the EFID trigger track reconstruction time on the mean number of pileup interactions using $\mathrm{Z} \rightarrow \mu^{+} \mu^{-}$Monte Carlo simulated data. Shown are the times for the most time-consuming tracking parts the main pattern recognition algorithm (circles) and Ambiguity Solver (squares) together with the total EFID tracking time (solid points) [6].

Additionally the positioning of the full Event Building in the full processing chain is more flexible, and can be deferred until much later in the processing chain.

The new HLT processing has two parts. The first algorithm handles the pattern recognition and is known as the Fast Track Finder (FTF). The pattern recognition forms a track based on spacepoint triplets across the layers in the ID. The FTF is designed to generate medium quality tracks as quickly as possible and to provide them as the seeds for the subsequent Precision Tracking (PT) algorithm. The PT is offline-like algorithm optimised for speed. It resolves the hit to track association ambiguities and removes duplicate tracks via tracks scoring to finally perform accurate track fitting.

The modification in the HLT tracking took also into account a new hardware components which will prepend HLT, namely the FTK [9]. That component will reconstruct tracks using massively parallel processing for every event accepted by Level 1 and these tracks will be available at early stage of HLT processing. HLT tracking software is prepared to be seeded by tracks from FTK which will be deployed in 2016.

\section{OPTIMISATIONS FOR RUN 2}

The architectural changes of the ATLAS trigger were complemented by code optimisations as well. The upgrade provided an opportunity to optimise the old code and write the new one to be efficient from start.

The optimisation was initiated by the identification of inefficient parts of the ATLAS trigger code - referred to as hot spots - which were target of optimisations. These most often called, or computationally expensive parts of the code were determined using a profiling tool Valgrind [10]. Other optimisations, such as including modifications to avoid branch misprediction or cache misses, have also been studied previously [11]. The architectural changes, optimisations, faster 


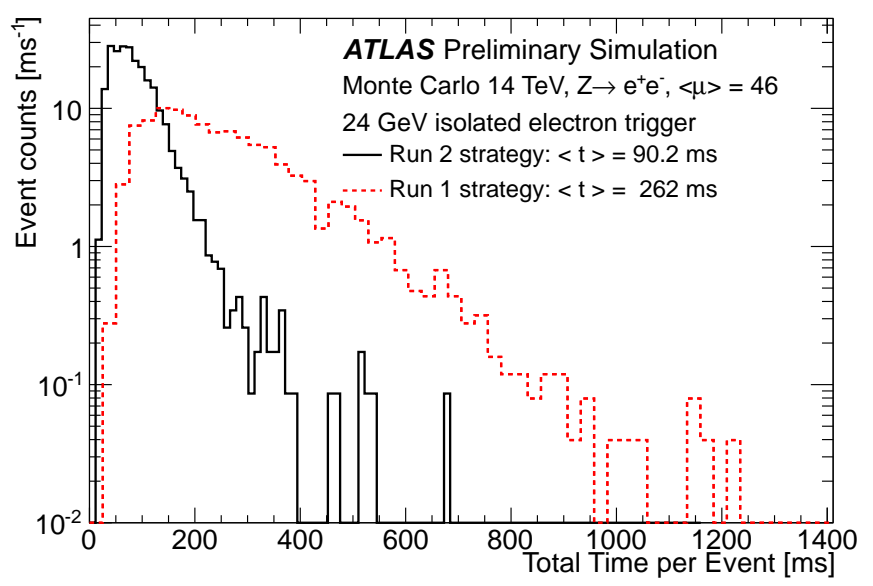

Fig. 3. The distribution of total processing time of the electron trigger is shown. The plot compares the tracking strategy that was executed during Run 1 (dashed red line) to the new Run 2 strategy (solid black line) both running on $\mathrm{Z} \rightarrow \mathrm{e}^{+} \mathrm{e}^{-}$Monte Carlo simulated data [6].

linear algebra library, etc. contributed to threefold reduction of the execution time of the Run 2 ATLAS trigger tracking code as shown in Fig. 3 in comparison with the Run 1 strategy on simulated Run 2 data in 2014. The detailed timing study of the Run 2 HLT ID tracking code is described in Ref. [12].

\section{2-STAGE TRACKING IN TAU RECONSTRUCTION}

After the upgrade, the new architecture is more flexible and facilitates new tracking strategies that can be more efficient and decrease the execution time in specific use cases.

The Run 1 tau reconstruction chain executed one large RoI so that is able to locate mostly hadronically decaying tau particle - the only lepton that is capable of this type of decay. The RoI was extended along the beam direction in shape $\Delta \eta=0.4, \Delta \phi=0.4$, and $\Delta z=225 \mathrm{~mm}$ with respect to the RoI direction and position $z=0$, to be able to reconstruct all the products of decaying tau.

A new tracking strategy for the tau chain in Run 2 processes RoIs in two phases to reduce the execution time by reducing the RoI volume. The first stage task is to find the leading particle track with the highest transverse momentum along the beam. Although the first step RoI is spread along the $\mathrm{z}$ coordinate the same way as in the old strategy it can be less open, such as $\Delta \eta=0.1, \Delta \phi=0.1$, and $\Delta z=225 \mathrm{~mm}$. Since the leading track is located by the first stage, the second stage can reconstruct the surrounding tracks in the wider region around it using RoI with the following parameters $\Delta \eta=0.4$, $\Delta \phi=0.4$, and with $\Delta z=10 \mathrm{~mm}$ only. Both strategies are compared with respect to the RoI sizes in Fig. 6. One can clearly see the total RoI volume in case of the new strategy is much smaller despite the overlapping RoIs.

CPU timing for both strategies is depicted in Fig. 4 for FTF where the data from RoIs are processed and then for the PT in Fig. 5. The new two-step tracking strategy is one third faster since the total mean processing time of the old single-stage strategy is $78 \mathrm{~ms}$ and the new two-step strategy is $49 \mathrm{~ms}$. The timing study was performed on the latest Run 2 data from August 2015.

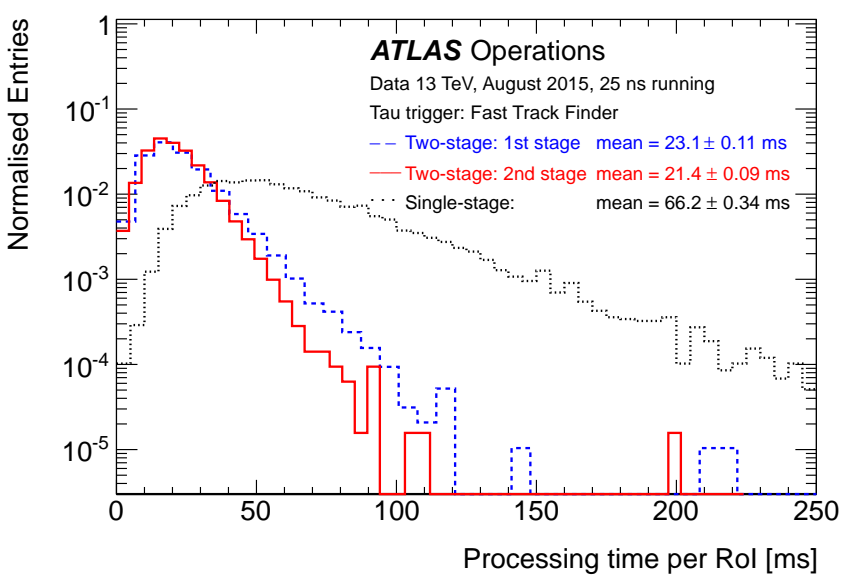

Fig. 4. The Run 2 HLT ID tracking trigger processing time for the FTF stage for the tau signature. Comparison between the single-stage (Run 1 configuration), and the two-stage (Run 2 configuration) tracking running on the Run 2 data [6].

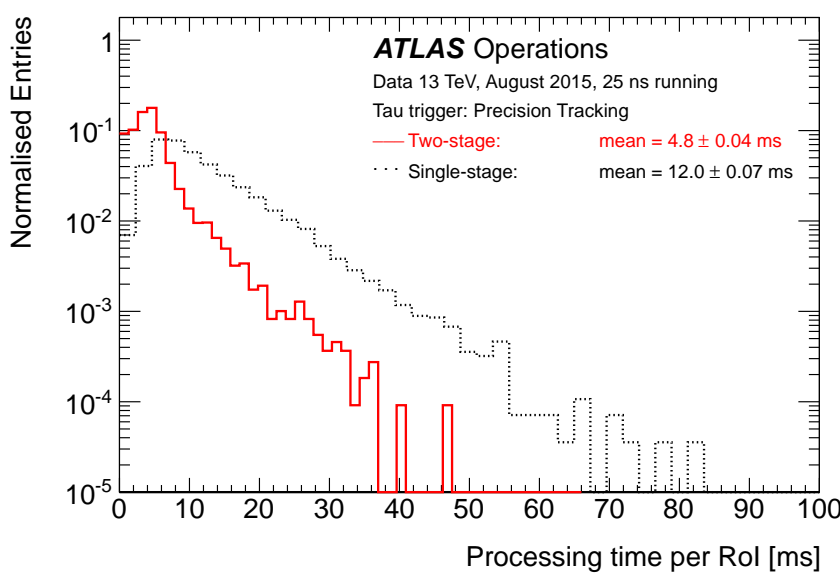

Fig. 5. The Run 2 HLT ID tracking trigger processing time for the PT stage for the tau signature. Comparison between the single-stage (Run 1 configuration), and the two-stage (Run 2 configuration) tracking running on the Run 2 data [6].

The same feature described in this section can be used for the other signatures such as the muon or electron isolation, $\mathrm{b}$-jets vertexing, and precision b-tagging.

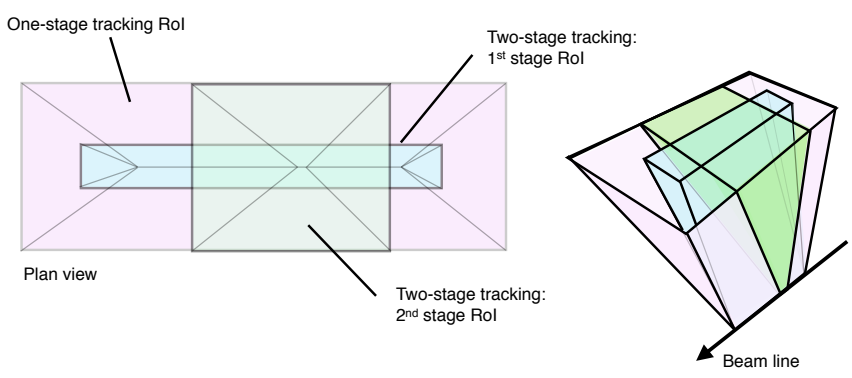

Fig. 6. An illustration of the RoI arrangement from the one-stage tracking (biggest RoI) and two-stage tracking (tightest RoI - first stage, squared RoI second stage) [6]. 


\section{RUN 2 ATLAS TRIGGER PERFORMANCE}

The ATLAS ID trigger is a key component of the HLT that has to make a decision whether to keep the collision event recorded by the ATLAS stored or not in a very short time period. The trigger performance is commonly measured with respect to the offline tracking algorithm that has more time for the event reconstruction. The trigger reconstruction is also referred as the online reconstruction.

The efficiency of the trigger tracking algorithm is the fraction of the number of all reconstructed tracks by online algorithm that match with the offline reconstructed tracks over the all offline tracks. The closest matching trigger track within a cone of $\Delta R<0.05$ to the offline track is chosen. The efficiency as a function of pseudorapidity $(\eta)$ and transverse momentum $\left(p_{\mathrm{T}}\right)$ is shown in Fig. 7 and 8 for muon chain and in Fig. 9 and 10 for electron chain. The efficiencies are shown for both trigger stages the FTF and PT. The efficiency of the muon trigger is almost $100 \%$ in the first Run 2 data which

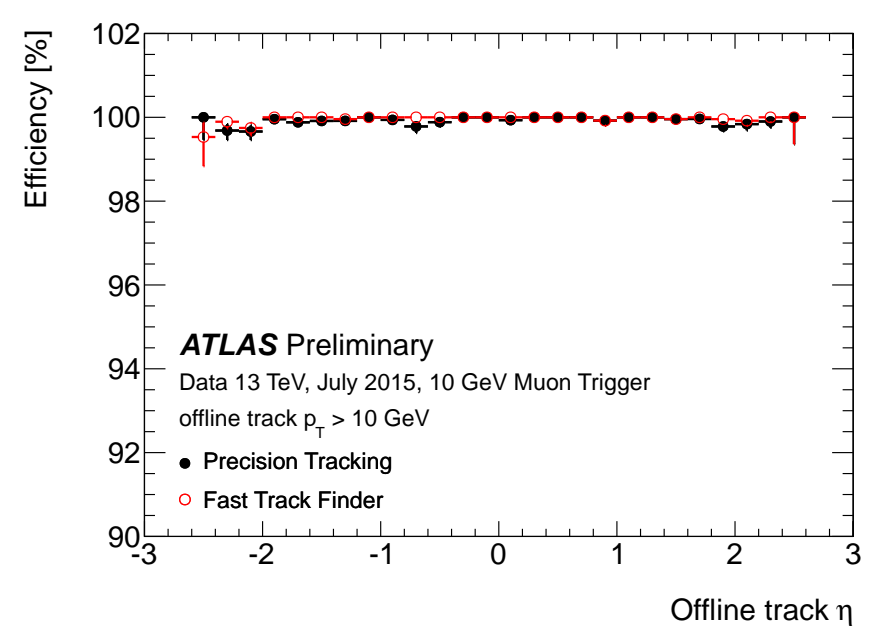

Fig. 7. The HLT ID tracking efficiency in the muon trigger as a function of pseudorapidity with respect to the offline muon candidate. Measured on the earliest Run 2 data for FTF and PT stage [6].

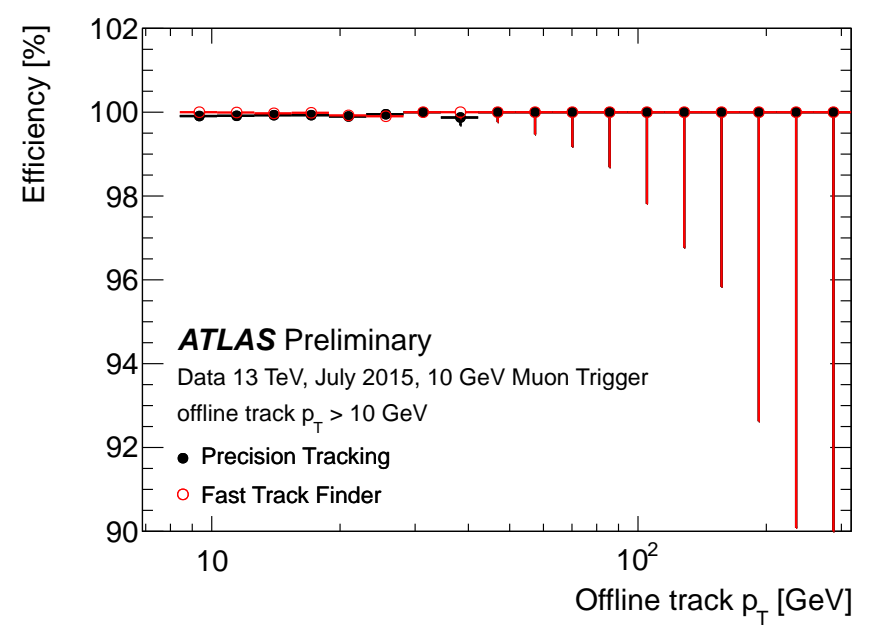

Fig. 8. The HLT ID tracking efficiency in the muon trigger as a function of transverse momentum $\left(p_{\mathrm{T}}\right)$ with respect to the offline muon candidate. Measured on the earliest Run 2 data for FTF and PT stage [6].

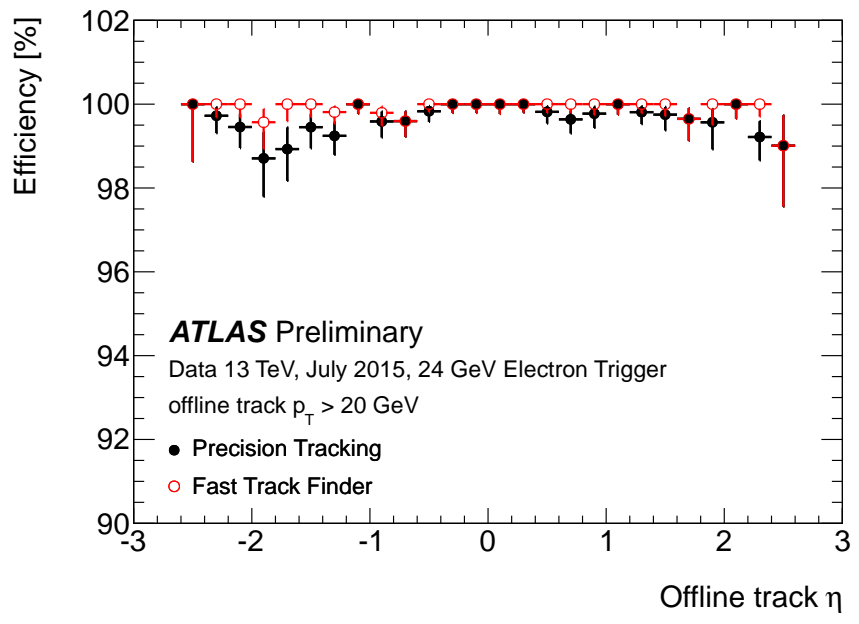

Fig. 9. The HLT ID tracking efficiency in the electron trigger as a function of pseudorapidity with respect to the offline electron candidate. Measured on the earliest Run 2 data for FTF and PT stage [6].

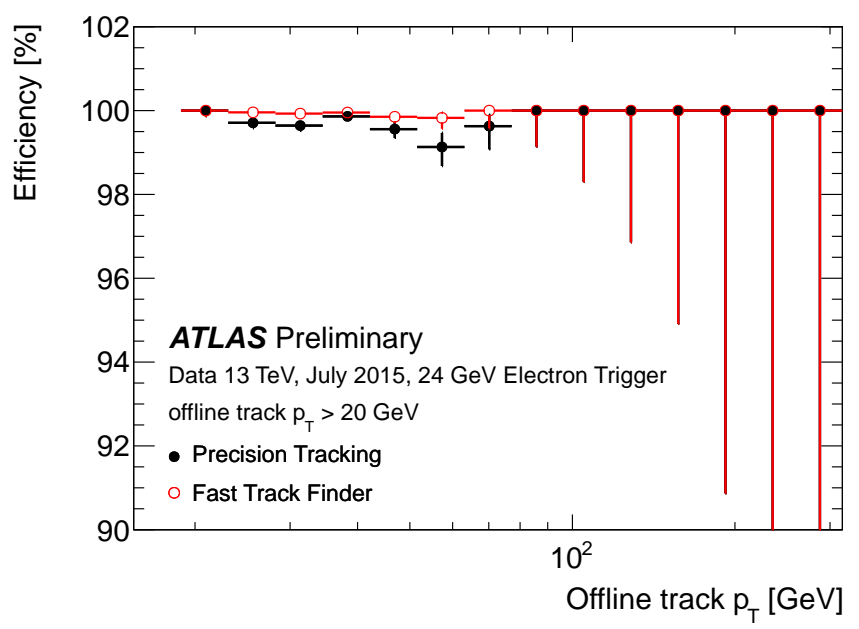

Fig. 10. The HLT ID tracking efficiency in the electron trigger as a function of transverse momentum $\left(p_{\mathrm{T}}\right)$ with respect to the offline electron candidate. Measured on the earliest Run 2 data for FTF and PT stage [6].

confirms the efficiency is as good as in Run 1. The electron trigger efficiency is higher than $99 \%$ and again is similar to the Run 1 efficiency. The FTF efficiency is slightly higher due to looser track requirements than in the PT which rejects the tracks of worse quality in the final stage.

Another measure of tracking quality is the difference between parameters of the tested online track and reference offline track called residual. The residual in the inverse $p_{\mathrm{T}}$ between the ID trigger track and the offline track for the muon trigger is shown in Fig. 11 and for the electron trigger in Fig. 12. This kind of residual is a measure of accuracy in momentum measurement and is proportional to track curvature. One can notice a positive tail in case of electrons due to energy loss from bremsstrahlung corrected for in the offline reconstruction algorithm. Both plots show the residuals are minimal with respect to the $p_{\mathrm{T}}$ cut.

The HLT ID tracking resolution of the transverse impact parameter with respect to the beamline in the muon trigger 


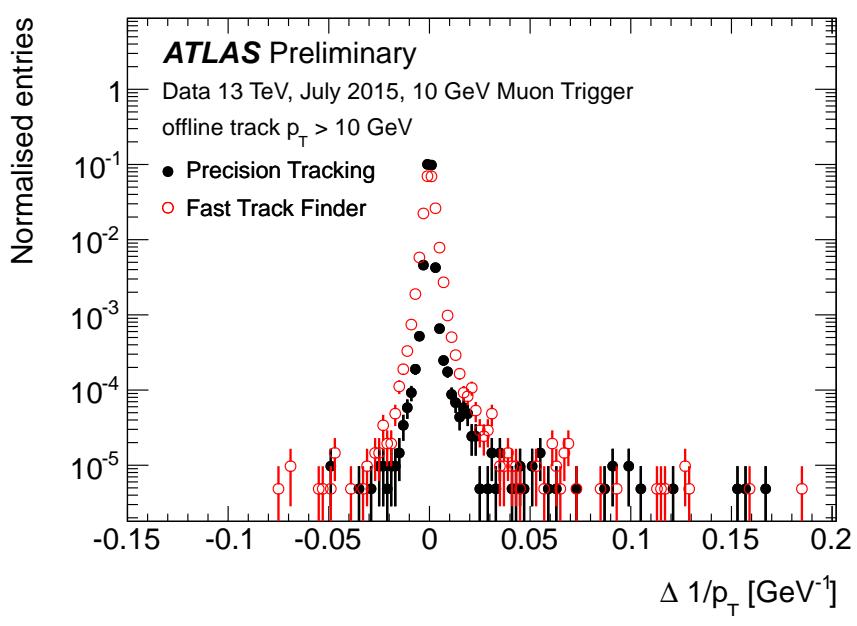

Fig. 11. The residual in inverse transverse momentum $\left(p_{\mathrm{T}}\right)$ between the HLT ID trigger track and the offline muon candidate track passing the muon trigger [6].

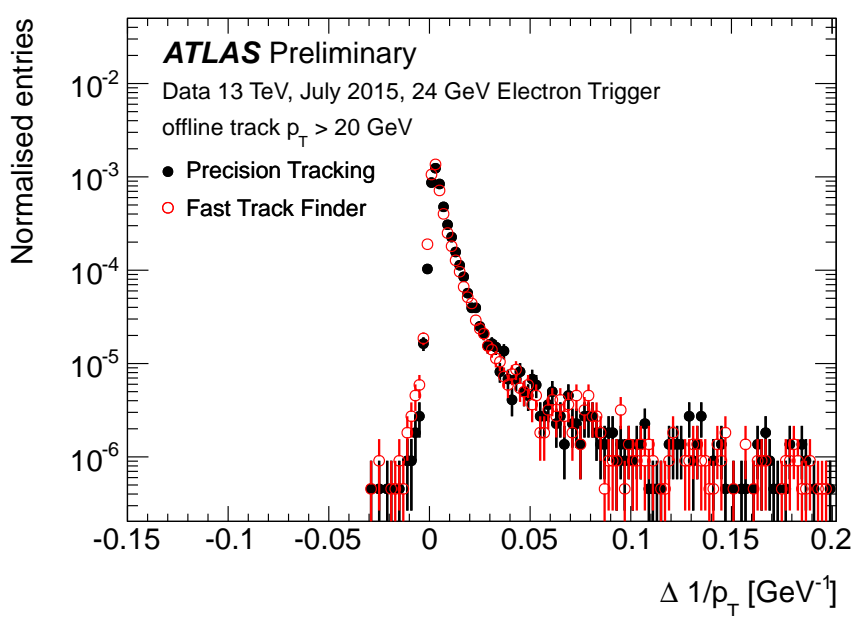

Fig. 12. The residual in inverse transverse momentum $\left(p_{\mathrm{T}}\right)$ between the HLT ID trigger track and the offline electron candidate track passing the electron trigger [6].

shown as a function of the pseudorapidity of the muon track for offline muon candidates can be seen in Fig. 13. A similar resolution plot of the pseudorapidity is shown in Fig. 14. In both cases the PT performs better due to the accurate final fitting. Resolution as a function of track pseudorapidity reflects an increasing amount of material due to geometry of the detector. Both plots show that the resolution is similar to the Run 1.

The shown HLT ID tracking efficiencies on the Run 2 data are in good agreement with the efficiencies measured on the Monte Carlo simulated data from validation studies during the upgrade period in 2014 [12].

\section{SUMMARY}

The ATLAS trigger system underwent several upgrades to be able to fulfill the new requirements given by enhanced parameters of the Run 2 LHC. The upgrade was done in a way to maintain the outstanding performance from Run 1 for the

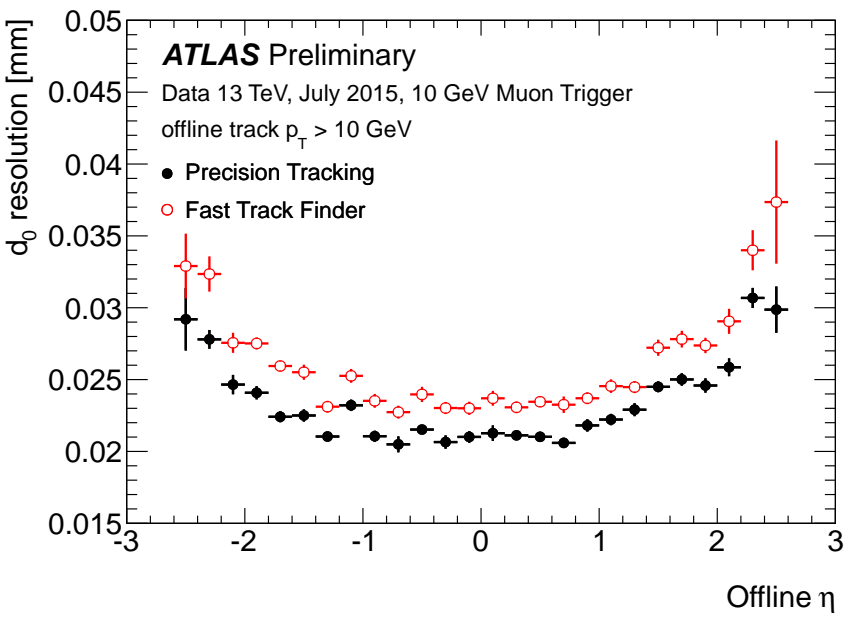

Fig. 13. The Run 2 HLT ID tracking resolution on the transverse impact parameter with respect to the beamline in the muon trigger shown as a function of the pseudorapidity of the muon track with respect to the offline muon candidates [6].

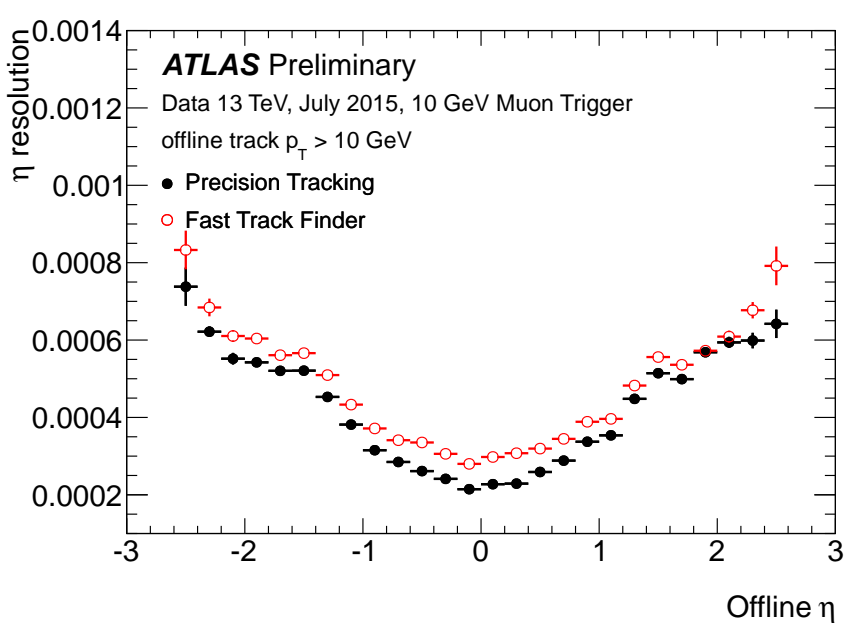

Fig. 14. The Run 2 HLT ID tracking resolution on the pseudorapidity with respect to the beamline in the muon trigger shown as a function of the pseudorapidity of the muon track with respect to the offline muon candidates [6].

next operation period. The trigger architecture was simplified to be more flexible and now provides new options improving the reconstruction process in the ATLAS HLT, such as the twostep tracking. Extensive profiling studies and optimisations were performed on the ATLAS tracking software to speed up the trigger decision process.

The paper shows the ATLAS ID trigger performance is in good shape for Run 2 and will help to extend the exceptional achievements of the detector from the first years of its operation.

\section{REFERENCES}

[1] ATLAS Collaboration, "Observation of a new particle in the search for the standard model higgs boson with the atlas detector at the lhc," Physics Letters B, vol. 716, no. 1, pp. 1 - 29, 2012. [Online]. Available: http://www.sciencedirect.com/science/article/pii/S037026931200857X

[2] P. Higgs, "Broken symmetries, massless particles and gauge fields," Physics Letters, vol. 12, no. 2, pp. 132 - 133, 1964. [Online]. Available: http://www.sciencedirect.com/science/article/pii/0031916364911369 
[3] F. Englert and R. Brout, "Broken symmetry and the mass of gauge vector mesons," Phys. Rev. Lett., vol. 13, pp. 321-323, Aug 1964. [Online]. Available: http://link.aps.org/doi/10.1103/PhysRevLett.13.321

[4] ATLAS Collaboration, "The ATLAS experiment at the CERN large hadron collider," Journal of Instrumentation, vol. 3, no. 08, p. S08003, 2008. [Online]. Available: http://stacks.iop.org/17480221/3/i=08/a=S08003

[5] ATLAS Collaboration, "Track Reconstruction Performance of the ATLAS Inner Detector at $\sqrt{s}=13 \mathrm{TeV}$," CERN, Geneva, Tech. Rep. ATLPHYS-PUB-2015-018, Jul 2015.

[6] ATLAS Collaboration. (2015, Oct.) HLT tracking public results. [Online]. Available: https://twiki.cern.ch/twiki/bin/view/AtlasPublic/ HLTTrackingPublicResults

[7] ATLAS Collaboration, "Performance of the ATLAS trigger system in 2010," The European Physical Journal C, vol. 72, no. 1, 2012. [Online]. Available: http://dx.doi.org/10.1140/epjc/s10052-011-1849-1

[8] R. Bartoldus et al., "Technical Design Report for the Phase-I Upgrade of the ATLAS TDAQ System," CERN, Geneva, Tech. Rep. CERNLHCC-2013-018. ATLAS-TDR-023, Sep 2013, final version presented to December 2013 LHCC.

[9] M. Shochet, et al., "Fast TracKer (FTK) Technical Design Report," CERN, Geneva, Tech. Rep. CERN-LHCC-2013-007. ATLAS-TDR-021, Jun 2013, ATLAS Fast Tracker Technical Design Report.

[10] N. Nethercote and J. Seward, "Valgrind: A framework for heavyweight dynamic binary instrumentation," in Proceedings of the 2007 ACM SIGPLAN Conference on Programming Language Design and Implementation, ser. PLDI '07. New York, NY, USA: ACM, 2007, pp. 89-100. [Online]. Available: http://doi.acm.org/10.1145/1250734.1250746

[11] ATLAS Collaboration, "Studies for the development of the Inner Detector trigger algorithms at ATLAS," CERN, Geneva, Tech. Rep. ATLDAQ-PUB-2013-002, Sep 2013.

[12] O. Penc and ATLAS Collaboration, "The performance and development for the inner detector trigger algorithms at ATLAS," Journal of Physics: Conference Series, vol. 608, no. 1, p. 012008, 2015. [Online]. Available: http://stacks.iop.org/1742-6596/608/i=1/a=012008 